\title{
Combined use of drugs inhibiting the renin- angiotensin system: prescribing patterns and risk of acute kidney injury in German nursing home residents
}

This article was published in the following Dove Press journal:

Clinical Interventions in Aging

\author{
Michael Dörks' \\ Stefan Herget-Rosenthal ${ }^{2}$ \\ Falk Hoffmann' \\ Kathrin Jobski' \\ 'Department of Health Services \\ Research, Carl von Ossietzky \\ University Oldenburg, Oldenburg, \\ Germany; ${ }^{2}$ Department of Medicine, \\ Rotes Kreuz Krankenhaus, Bremen, \\ Germany
}

Correspondence: Michael Dörks

Department of Health Services Research, Carl von Ossietzky University Oldenburg, Ammerländer Heerstrasse I40,

26III Oldenburg, Germany

Tel +49 44l 7982890

Email michael.doerks@uni-oldenburg.de
Background/aims: In 2012, the European Medicines Agency reviewed the safety of dual renin-angiotensin system (RAS) blockade because of potentially increased risks for inter alia acute kidney injury (AKI). Since residents of nursing homes are particularly vulnerable to adverse drug outcomes, the aims of our study were to describe RAS-inhibiting drug use in German nursing home residents and examine the risk of AKI associated with dual RAS blockade.

Methods: Based on claims data, a nested case-control study within a cohort of RAS-inhibiting drug users was conducted. Using conditional logistic regression, confounder-adjusted odds ratios (aORs) and 95\% confidence intervals (CI) were obtained for the risk of AKI associated with dual RAS blockade. Subgroup analyses were performed in patients with diabetes or chronic kidney disease and both comorbidities.

Results: Of all 127,227 nursing home residents, the study cohort included 64,567 (50.7\%) who were treated with at least one RAS-inhibiting drug. More than three quarters of the study population were female (77.1\%). Mean age was $86.0 \pm 6.8$ years. Most residents were treated with angiotensin-converting enzyme inhibitors $(77.8 \%)$, followed by angiotensin II receptor blockers $(21.6 \%)$ and aliskiren $(0.2 \%)$. Annual prevalence of dual RAS blockade declined from 9.6 (95\% CI 7.8-11.8) in 2010 to 4.7 (95\% CI 4.0-5.4) per 1,000 users in 2014. In the overall cohort, AKI was not significantly associated with dual RAS blockade (aOR 1.99; 0.77-5.17). However, significantly increased aORs were observed when considering patients with diabetes (3.47; 1.27-9.47), chronic kidney disease $(4.74 ; 1.24-18.13)$ or both $(11.17 ; 2.65-47.15)$.

Conclusions: Prescribing of drugs inhibiting the RAS is common in German nursing homes. Though the prevalence of dual RAS blockade declined, our study showed an increased risk of AKI in patients with diabetes and/or chronic kidney disease. Therefore, cautious use is warranted in these vulnerable patients.

Keywords: dual RAS blockade, nested case-control study, long-term care

\section{Introduction}

All three classes of available renin-angiotensin system (RAS) inhibitors, angiotensinconverting enzyme inhibitors (ACEIs), angiotensin II receptor blockers (ARBs), and the direct renin inhibitor aliskiren interrupt the normal angiotensin II feedback suppression of renin secretion from the kidneys. Previous data suggested that dual RAS blockade may have more positive effects on cardiovascular endpoints and development of severe chronic kidney disease than RAS blockade by a single agent. ${ }^{1,2}$ However, in several large randomized controls trials and a meta-analysis dual RAS blockade was 
associated with an excessive risk of acute kidney disease (AKI), hyperkalemia, hypotension and dialysis. ${ }^{3-6}$ Based on these studies, the European Medicines Agency (EMA) issued new contraindications and warnings for aliskirencontaining drugs in 2012 and endorsed restrictions on the combined use of ARBs, ACEIs and direct renin inhibitors in 2014. ${ }^{7}$ ACEIs and ARBs should not be used concomitantly in patients with diabetic nephropathy and the use of aliskiren with an ARB or an ACEI is contraindicated in patients with diabetes or chronic kidney disease stage 3-5 (glomerular filtration rate $[\mathrm{GFR}]<60 \mathrm{~mL} / \mathrm{min} / 1.73 \mathrm{~m}^{2}$ ). However, use of candesartan or valsartan as add-on therapy to an ACEI is still licensed in patients with heart failure intolerant to mineralocorticoid antagonists and with persistent symptoms despite other therapies. ${ }^{7}$

In Germany, about 800,000 people live in nursing homes. ${ }^{8}$ This population is typically characterized by a high prevalence of chronic diseases including diabetes and chronic kidney disease. ${ }^{9-15}$ The prevalence of chronic kidney disease stage $3-5$ in nursing home residents is between $44 \%$ and $63 \% .{ }^{10}$ Prescribing of drugs is a crucial indicator of quality of care in nursing homes, ${ }^{16}$ since residents of nursing homes are treated with more drugs than older people living in their own homes, and half of them take at least one inappropriate medication. ${ }^{17,18}$ Therefore, residents of nursing homes are particularly vulnerable to adverse drug outcomes as a consequence of inappropriate drug use..$^{11,12,14,19,20}$ Likewise, an impaired kidney function is a key safety issue associated with dual RAS blockade that may lead to AKI. ${ }^{7}$

Against this background, the aims of our study were 1) to describe RAS-inhibiting drug use, especially dual RAS blockade, in German nursing home residents, 2) to assess potential changes of prescribing prevalence over time and 3) to examine the risk of AKI associated with dual RAS blockade compared with the use of one RAS-inhibiting drug class.

\section{Materials and methods Data source and study design}

Using claims data from a large statutory health insurance fund insuring about six million persons (over seven percent of the German population), this cohort study was based on new nursing home residents from 1 January 2010 to 31 December 2014 aged 65 years or older who had been continuously insured in the year preceding nursing home entry. For each resident sociodemographic data, information on in- and outpatient diagnoses as well as outpatient drug prescriptions (including the number of prescribed defined daily doses [DDDs]) and care level were available. Diagnoses were based on the German modification of the International Classification of Diseases, 10th revision (ICD-10-GM). Inpatient diagnoses could be referred to an exact date, while outpatient diagnoses were coded on a quarterly basis (ie, a 3-month interval). Additionally, information includes the type of diagnosis (main/ancillary/secondary) for inpatient and the diagnostic certainty (confirmed/suspected/ruled out/ status post) for outpatient diagnoses.

Patients were enrolled in the study cohort if they had filled at least one outpatient prescription of an RAS-inhibiting drug during nursing home stay. The date of the first RAS-inhibiting drug prescription was defined as cohort entry. Cohort exit was set as the first of the following dates: 1) end of study period, 2) death from any cause or 3) deregistration from the insurance provider. For the risk study, hospitalization for AKI and the end of the first continuous treatment with RAS-inhibiting drugs were additionally defined as cohort exit.

A nested case-control study was conducted to assess the risk of AKI associated with dual blockade compared with the use of one RAS-inhibiting drug class alone. Within the cohort of patients continuously treated with at least one RAS-inhibiting drug, up to 20 controls were matched to each case hospitalized for AKI with respect to year of birth and sex using risk-set sampling. An index date was assigned to each control resulting in the same length of follow-up after nursing home admission as for the corresponding case. Patients might have served as controls for more than one case and were eligible to be selected as controls until they became a case.

Patient informed consent was not required by law as the study was based on anonymous data.

\section{Drug exposures}

Analyzed drugs were classified based on the seventh level of the Anatomical-Therapeutic-Chemical (ATC) code. Drug classes were considered based on the fourth level of the ATC code. The following drug classes were included in the analyses: ACEIs (ATC code C09A), ARBs (ATC code C09C), including fixed combinations with other drugs such as diuretics (ATC codes C09B and C09D, respectively) and the direct renin inhibitor aliskiren (ATC code C09XA) including combinations. Dual RAS blockade was defined as prescriptions of at least two different RAS-inhibiting drug classes on the same day. Nursing home residents who had not received an RAS-inhibiting agent in the 12 months preceding the index prescription were considered as incident users. Potential switching of RAS-inhibiting drug classes after nursing home entry was analyzed by considering the last prescription before 
and the first prescription after nursing home admission within a 365-day period. For these analyses, only prevalent users were considered. The specialty of the prescribing physician was analyzed with respect to prescriptions by general practitioners, nephrologists, cardiologists, other specialists for internal medicine and other miscellaneous specialties. The duration of each prescription was estimated assuming that a patient's daily dose was one DDD. Allowing a grace period of 7 days, subsequent prescriptions were considered as continuous treatment.

\section{Outcome}

AKI (ICD-10-GM code N17) was assessed in hospital main discharge diagnoses. The time of the event was defined as the date of hospital admission.

\section{Covariates}

Baseline covariates were assessed in the quarter of cohort entry. Potential indications and comorbidity were obtained from confirmed outpatient diagnoses: chronic kidney disease (defined as ICD-10-GM codes N18, N19), heart failure (ICD10-GM code I50), diabetes (ICD-10-GM code E10-E14) and hypertension (ICD-10-GM code I10-I15). Equally, the number of different drugs (based on the seventh level of the ATC code) other than RAS-inhibiting agents was assessed during this time period. Following previous studies, polypharmacy was defined as prescribing of five or more long-term medications and grouped into treatment with 5-9 (polypharmacy) and 10 or more drugs (excessive polypharmacy). ${ }^{15}$ The care level was assessed on the day of cohort entry ranging from 0/I (limited daily living skills/substantial need of care) to III (most severe need of care).

For the case-control study, the following covariates were assessed from confirmed outpatient diagnoses in the quarter preceding the quarter of the index date: heart failure, diabetes, chronic kidney disease and hypertension. Further, the care level, polypharmacy and excessive polypharmacy (assessed in the quarter preceding the quarter of the index date) were included. Prescriptions of drugs defined as dual RAS blockade were assessed from outpatient dispensations during the 30 days before the index date.

\section{Statistical analysis}

Baseline data were analyzed using descriptive statistics. Age at the time of cohort entry was stratified according to the following classes: $<70,70-79,80-89$ and $\geq 90$ years. Quarterly and annual prevalences of dual RAS blockade were calculated by dividing the number of patients treated with dual RAS blockade by the number of patients receiving at least one RAS-inhibiting drug during the respective time period. Two-sided 95\% CIs were estimated. ${ }^{21}$ Conditional logistic regression was conducted to obtain confounder-adjusted odds ratios (aORs) with corresponding 95\% CIs for the risk of AKI associated with dual RAS blockade.

All statistical analyses were performed with SAS statistical software version 9.4 (SAS Institute, Cary, NC, USA).

\section{Sensitivity and subgroup analyses}

In sensitivity analyses, dual RAS blockade was defined as prescriptions of at least two different RAS-inhibiting drug classes in a 7-, 14- and 30-day period. Subgroup analyses were based on 1) patients with diabetes, 2) chronic kidney disease and 3 ) the combination of both comorbidities, respectively.

\section{Ethical approval}

The use of anonymous data for this study was approved by the involved statutory health insurance. Informed consent and approval by an ethics committee were not required.

\section{Results \\ Use of drugs inhibiting the RAS}

Of all 127,227 nursing home residents, the study cohort included 64,567 (50.7\%) who were treated with at least one RAS-inhibiting agent and, therefore, were solely considered (Table 1). More than three quarters of the study population were female $(77.1 \%)$. Mean age was $86.0 \pm 6.8$ years (interquartile range: 80-90). Commonly diagnosed diseases were hypertension $(85.2 \%)$, diabetes $(34.2 \%)$ and heart failure (29.9\%). About half of all patients of the study cohort (51.8\%) were treated with 5-9, and more than one third (33.9\%) with 10 or more different drugs. Based on the first prescription during their nursing home stay, most cohort patients were treated with ACEIs (77.8\%), followed by ARBs $(21.6 \%)$ and aliskiren $(0.2 \%)$, while $0.4 \%$ received two or three different RAS-inhibiting drug classes on that day. Prescribing specialties were general practitioners $(97.0 \%)$, nephrologists $(0.3 \%)$ and other specialists for internal medicine $(1.0 \%)$. Dual RAS blockade was prescribed by general practitioners $(96.0 \%)$, cardiologists $(0.1 \%)$ and other specialists for internal medicine (1.0\%) (data not shown). Incident use of RAS-inhibiting drugs was found in 9,794 patients of the study cohort. In most of these patients, therapy was initiated with single use of ACEIs (90.3\%), followed by ARBs (9.4\%) and 
Table I Characteristics of nursing home residents treated with drugs inhibiting the RAS

\begin{tabular}{|c|c|c|c|c|c|}
\hline Study population ${ }^{a}$ & $<70$ years & 70-79 years & $80-89$ years & $>89$ years & Total \\
\hline Total & $\mathrm{I}, 63 \mathrm{I}(2.5 \%)$ & 12,474 (19.3\%) & 34,049 (52.7\%) & $16,4 \mid 3(25.4 \%)$ & $64,567(100 \%)$ \\
\hline \multicolumn{6}{|l|}{ Sex } \\
\hline Male & 711 (43.6\%) & 3,893 (31.2\%) & 7,524 (22.1\%) & $2,686(16.4 \%)$ & $|4,8| 4(22.9 \%)$ \\
\hline Female & $920(56.4 \%)$ & 8,581 (68.8\%) & 26,525 (77.9\%) & I3,727 (83.6\%) & $49,753(77.1 \%)$ \\
\hline \multicolumn{6}{|l|}{ Care level $^{\mathrm{b}}$} \\
\hline $0 / 1$ & $873(53.5 \%)$ & 6,902 (55.3\%) & $21,383(62.8 \%)$ & $10,249(62.4 \%)$ & 39,407 (61.0\%) \\
\hline II & $638(39.1 \%)$ & $4,590(36.8 \%)$ & $10,874(31.9 \%)$ & $5,45 \mathrm{I}(33.2 \%)$ & $21,553(33.4 \%)$ \\
\hline III & $120(7.4 \%)$ & $982(7.9 \%)$ & I,792 (5.3\%) & $7 \mid 3(4.3 \%)$ & 3,607 (5.6\%) \\
\hline \multicolumn{6}{|l|}{ Polypharmacyc } \\
\hline Non-polypharmacy & $160(9.8 \%)$ & I,368 (I I.0\%) & $4,605(13.5 \%)$ & $3,056(18.6 \%)$ & $9,189(14.2 \%)$ \\
\hline \multicolumn{6}{|l|}{$(<5$ drugs $)$} \\
\hline Polypharmacy & 738 (45.3\%) & $5,942(47.6 \%)$ & I7,793 (52.3\%) & $8,992(54.8 \%)$ & 33,465 (5I.8\%) \\
\hline \multicolumn{6}{|l|}{ (5-9 drugs) } \\
\hline Excessive polypharmacy & 733 (44.9\%) & 5,164 (4I.4\%) & $|I, 65|(34.2 \%)$ & $4,365(26.6 \%)$ & $21,913(33.9 \%)$ \\
\hline \multicolumn{6}{|l|}{$(\geq 10$ drugs $)$} \\
\hline \multicolumn{6}{|l|}{ RAS-inhibiting drugs ${ }^{d}$} \\
\hline Angiotensin-converting enzyme inhibitors & $\mathrm{I}, 300(79.7 \%)$ & $9,623(77.1 \%)$ & $26,383(77.5 \%)$ & 12,944 (79.9\%) & $50,250(77.8 \%)$ \\
\hline Angiotensin II receptor blockers & $317(19.4 \%)$ & $2,740(22.0 \%)$ & $7,476(22.0 \%)$ & $3,400(20.7 \%)$ & $13,933(21.6 \%)$ \\
\hline Aliskiren & $0(0.0 \%)$ & $27(0.2 \%)$ & $70(0.2 \%)$ & $31(0.2 \%)$ & $128(0.2 \%)$ \\
\hline Dual/triple RAS blockade & 14 (0.9\%) & $84(0.7 \%)$ & $120(0.4 \%)$ & $38(0.2 \%)$ & $256(0.4 \%)$ \\
\hline \multicolumn{6}{|l|}{ Comorbidity ${ }^{e}$} \\
\hline Hypertension (II0-15) & I,34I (82.2\%) & $10,533(84.4 \%)$ & $29,042(85.3 \%)$ & $14,038(85.5 \%)$ & $54,954(85.1 \%)$ \\
\hline Heart failure $(150)$ & $269(16.5 \%)$ & $2,518(20.2 \%)$ & $10,244(30.1 \%)$ & $6,232(38.0 \%)$ & $19,263(29.8 \%)$ \\
\hline Diabetes mellitus (EI0-I4) & $720(44.1 \%)$ & $5,201(41.7 \%)$ & 11,610 (34.1\%) & $4,536(27.6 \%)$ & 22,067 (34.2\%) \\
\hline Chronic kidney disease (NI8, NI9) & $265(16.3 \%)$ & $2,320(18.6 \%)$ & $6,984(20.5 \%)$ & 3,588 (21.9\%) & $13,157(20.4 \%)$ \\
\hline
\end{tabular}

Notes: ${ }^{a}$ Nursing home residents with at least one prescription of an RAS-inhibiting drug during nursing home stay, percentages except total numbers based on column attributes, age assessed at first RAS-inhibiting drug prescription after nursing home entry. 'Level of care at first RAS-inhibiting drug prescription after nursing home entry.

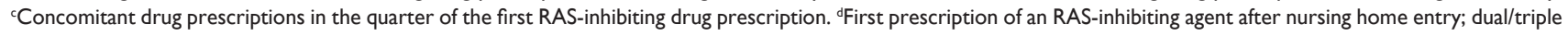

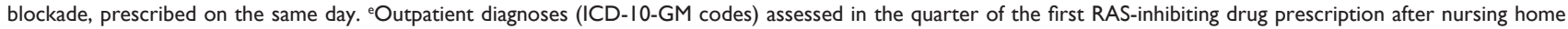
entry; patients could contribute to more than one line. 0/l: limited daily living skills/substantial need of care; II: severe need of care; III: most severe need of care.

Abbreviation: RAS, renin-angiotensin system.

aliskiren $(0.1 \%)$, while $0.2 \%$ were initially treated with two or more RAS-inhibiting drugs (Table 2). The consideration of potential switching after nursing home entry showed that of all patients of the study cohort 52,559 (81.4\%) maintained on their RAS-inhibiting drug class. Only a small proportion switched their drug class with nursing home admission. The largest number changed from an ARB to an ACEI or, vice versa, from an ACEI to an ARB.

\section{Prevalence of dual RAS blockade}

During nursing home stay, 608 of the 127,227 residents were treated at least once with dual RAS blockade defined as two different RAS-inhibiting drug classes prescriptions on the same day, resulting in an overall prevalence of $4.8(95 \% \mathrm{CI}$ 4.4-5.2) per 1,000 residents. Referring to the study cohort, quarterly prevalence of dual RAS blockade was $7.6(95 \%$ CI 4.4-13.3) per 1,000 users of RAS-inhibiting drugs in the

Table 2 Switching of drugs inhibiting the RAS at nursing home admission

\begin{tabular}{llllll}
\hline $\begin{array}{l}\text { First prescription after } \\
\text { nursing home admission }\end{array}$ & ACEI & ARB & Aliskiren & $\begin{array}{l}\text { Dual RAS } \\
\text { blockade }\end{array}$ & $\begin{array}{l}\text { Total } \\
\text { Total }\end{array}$ \\
$\begin{array}{llll}\text { Last prescription before nursing home admission } \\
\text { Incident user }\end{array}$ & $\begin{array}{l}50,250(78.1 \%) \\
\text { ACEI }\end{array}$ & $13,933(21.7 \%)$ & $128(0.2 \%)$ & $256(0.4 \%)$ & $64,567(100 \%)$ \\
ARB & $40,847(17.6 \%)$ & $924(6.6 \%)$ & $7(5.5 \%)$ & $16(6.3 \%)$ & $9,794(15.2 \%)$ \\
Aliskiren & $1,094(2.2 \%)$ & $562(4.0 \%)$ & $19(14.8 \%)$ & $87(34.0 \%)$ & $40,797(63.2 \%)$ \\
Dual RAS blockade & $42(0.1 \%)$ & $12,293(88.2 \%)$ & $16(12.5 \%)$ & $73(28.5 \%)$ & $13,476(20.9 \%)$ \\
\hline
\end{tabular}

Note: ${ }^{a}$ Potential switching of RAS-inhibiting drug classes after nursing home entry was analyzed by considering the last prescription before and the first prescription after nursing home admission within a 365-day period.

Abbreviations: ACEI, angiotensin-converting enzyme inhibitors; ARB, angiotensin II receptor blockers; RAS, renin-angiotensin system. 


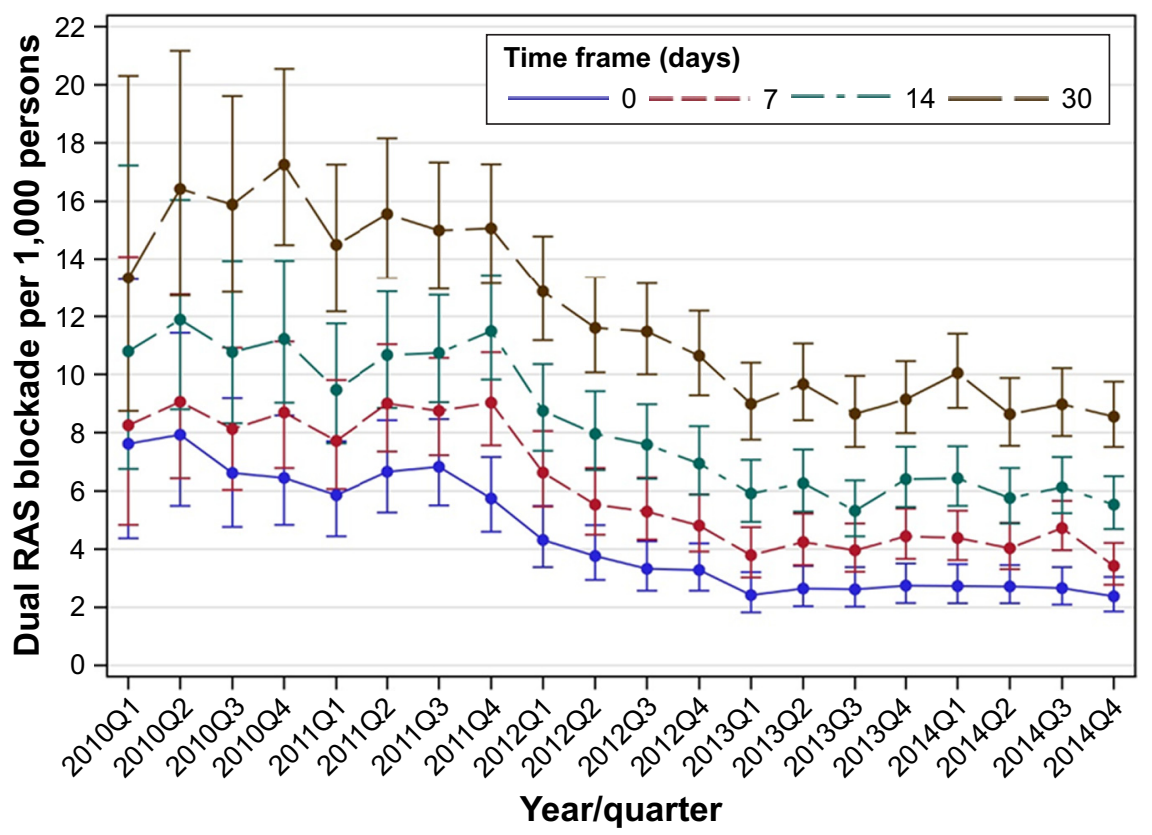

Figure I Prevalence of dual RAS blockade in nursing home residents during the study period.

Abbreviations: RAS, renin-angiotensin system; Q, quarter.

first quarter of 2010 (Figure 1). During the study period, the prevalence decreased to 2.4 (95\% CI 1.8-3.0) in the last quarter of 2014. In the sensitivity analyses, when the prevalence of dual RAS blockade was defined as prescriptions of at least two different RAS-inhibiting drug classes in a 7-, 14- and 30-day period, the prevalence per quarter increased in each case, ie, the larger the chosen time period, the larger the quarterly prevalence. The decrease during the study period was similar regardless of the underlying time period. Defined as concomitant prescriptions on the same day, annual prevalence of dual RAS blockade declined from 9.6 (95\% CI 7.8-11.8) in 2010 to 4.7 (95\% CI 4.0-5.4) per 1,000 users of RAS-inhibiting drugs in 2014 (data not shown).

In every considered study year, ACEIs and ARBs were most commonly combined as part of a dual RAS blockade (Figure 2). The proportion of aliskiren prescriptions as part

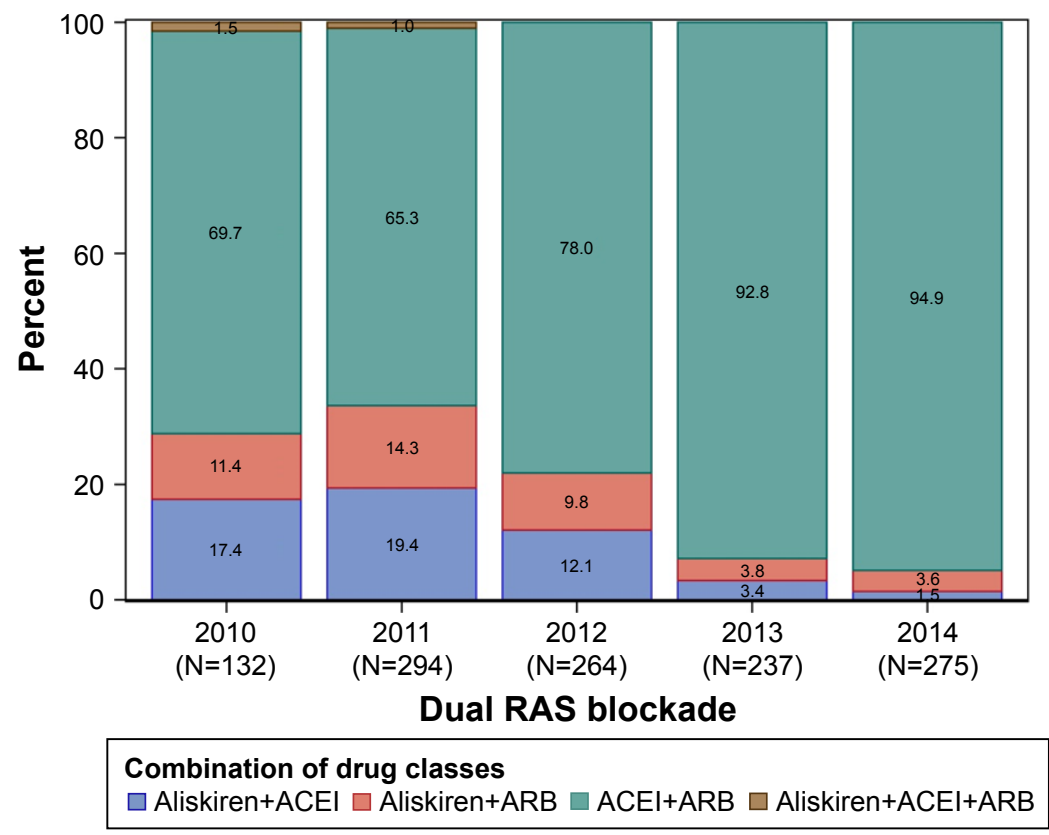

Figure 2 Combined use of drugs inhibiting the RAS in nursing home residents during the study period.

Abbreviations: ACEI, angiotensin-converting enzyme inhibitors; ARB, angiotensin II receptor blockers; RAS, renin-angiotensin system. 
Table 3 Acute kidney injury associated with dual RAS blockade in nursing home residents compared with the use of one RASinhibiting drug only

\begin{tabular}{|c|c|c|c|}
\hline & $\begin{array}{l}\text { Cases } \\
(\mathbf{N}=I, 037)\end{array}$ & $\begin{array}{l}\text { Controls } \\
(N=20,596)\end{array}$ & $\begin{array}{l}\text { Adjusted OR } \\
(95 \% \mathrm{Cl})\end{array}$ \\
\hline Dual RAS blockade ${ }^{a}$ & $5(0.5 \%)$ & $39(0.2 \%)$ & $1.99(0.77-5.17)$ \\
\hline Diabetes & 476 (45.9\%) & 7,291 (35.4\%) & $1.23(1.08-1.4 I)$ \\
\hline Heart failure & 399 (38.5\%) & $5,653(27.4 \%)$ & $1.33(1.16-1.52)$ \\
\hline Hypertension & $903(87.1 \%)$ & $17,523(85.1 \%)$ & $0.98(0.8 \mathrm{I}-\mathrm{I} .18)$ \\
\hline Chronic kidney disease & 406 (39.2\%) & $4,253(20.7 \%)$ & $2.03(1.78-2.33)$ \\
\hline Care level $0 / l^{b}$ & 605 (58.3\%) & $|2,40|(60.2 \%)$ & Ref \\
\hline Care level IIb & $384(37.0 \%)$ & $6,799(33.0 \%)$ & $1.09(0.96-1.25)$ \\
\hline Care level IIIb & $48(4.6 \%)$ & I,396 (6.8\%) & $0.70(0.52-0.95)$ \\
\hline Non-polypharmacy & $181(17.5 \%)$ & 6,433 (3I.2\%) & Ref \\
\hline \multicolumn{4}{|l|}{$(<5 \text { drugs })^{c}$} \\
\hline Polypharmacy & $519(50.0 \%)$ & $10,450(50.7 \%)$ & $1.59(1.33-1.89)$ \\
\hline \multicolumn{4}{|l|}{$(5-9 \text { drugs })^{c}$} \\
\hline Excessive & 337 (32.5\%) & 3,713 (I8.0\%) & 2.5 I (2.07-3.05) \\
\hline \multicolumn{4}{|l|}{ polypharmacy } \\
\hline$\left(\geq 10\right.$ drugs $^{c}$ & & & \\
\hline
\end{tabular}

Notes: ${ }^{\mathrm{a} D e f i n e d}$ as prescriptions of at least two different drug classes inhibiting the RAS on the same day. ${ }^{\text {b} A s s e s s e d ~ i n ~ t h e ~ q u a r t e r ~ p r e c e d i n g ~ t h e ~ q u a r t e r ~ o f ~ t h e ~}$ index date. 'Concomitant drug prescriptions (other than RAS-inhibiting drugs) in the quarter preceding the quarter of the index date. 0/l: limited daily living skills/ substantial need of care; II: severe need of care; III: most severe need of care.

Abbreviation: RAS, renin-angiotensin system.

of a dual RAS blockade declined over the study period, while concomitant prescribing of an ACEI and an ARB increased.

\section{Risk for AKI}

In the nested case-control study, 1,037 patients continuously treated with RAS-inhibiting drugs were identified who were admitted to hospital due to AKI (Table 3). In the overall cohort, AKI was not significantly associated with dual RAS blockade compared to being treated with one RASinhibiting drug class only (aOR 1.99, 95\% CI 0.77-5.17). Diabetes (aOR 1.23, 95\% CI 1.08-1.41), heart failure (aOR 1.33, 95\% CI 1.16-1.52), chronic kidney disease (aOR
2.03, 95\% CI 1.78-2.33), polypharmacy (aOR 1.59, 95\% CI 1.33-1.89) and excessive polypharmacy (aOR 2.51, 95\% CI 2.07-3.05) were associated with AKI in the multivariable analysis. Applying the 7-day, the 14-day or the 30-day period (Table 4), dual RAS blockade yielded aORs of 2.37 (95\% CI 1.05-5.32), 2.30 (95\% CI 1.13-4.67) and 2.03 (95\% CI $1.07-3.84)$, respectively.

Considering patients with diabetes, chronic kidney disease or both, significantly increased aORs for dual RAS blockade were observed irrespective of its underlying definition. With respect to nursing home residents with diabetes, the aOR of AKI was 3.47 (95\% CI 1.27-9.47) for prescriptions of at least two different RAS-inhibiting drug classes on the same day. For the 7-day, the 14-day and the 30-day windows, the aORs were 3.13 (95\% CI 1.26-7.78), 3.02 (95\% CI 1.31-6.96) and 3.01 (95\% CI 1.45-6.25). With respect to nursing home residents with chronic kidney disease, the aORs were 4.74 (95\% CI 1.24-18.13) for prescriptions on the same day and, respectively, 4.84 (95\% CI 1.54-15.19, 7-day period), 4.02 (95\% CI 1.49-10.87, 14-day period) and 3.67 (95\% CI 1.59-8.48, 30-day period). With regard to patients with both diabetes and chronic kidney disease, the resulting aORs were higher than the aORs for residents with solely diabetes or chronic kidney disease $(11.17[95 \% \mathrm{CI}$ 2.65-47.15, same day], 9.85 [95\% CI 2.85-34.02, 7-day period], 8.96 [95\% CI 3.01-26.67, 14-day period] 9.67 [95\% CI 3.81-24.59, 30-day period]).

\section{Discussion}

Prescribing of RAS-inhibiting agents, mainly ACEIs, is common in German nursing homes. In contrast, only a small proportion of nursing home residents receive dual RAS blockade. The prevalence of dual RAS blockade declined substantially over the study period. An increased risk for

Table 4 Acute kidney injury associated with dual RAS blockade in nursing home residents compared with use of one RAS-inhibiting drug only: subgroup analyses and sensitivity analyses based on different definitions of dual RAS blockade

\begin{tabular}{|c|c|c|c|c|}
\hline & \multicolumn{4}{|c|}{ Adjusted OR (95\% Cl) } \\
\hline & $\begin{array}{l}\text { All patients } \\
(N=21,633)\end{array}$ & $\begin{array}{l}\text { Patients with } \\
\text { diabetes }^{\mathrm{b}} \\
(\mathbf{N}=\mathbf{9 , 5 6 8 )}\end{array}$ & $\begin{array}{l}\text { Patients with chronic } \\
\text { kidney disease }^{c} \\
(\mathbf{N}=\mathbf{7 , 8 8 6})\end{array}$ & $\begin{array}{l}\text { Patients with diabetes and } \\
\text { chronic kidney disease }{ }^{d} \\
(N=3,947)\end{array}$ \\
\hline \multicolumn{5}{|c|}{ Dual RAS blockade ${ }^{e}$} \\
\hline On the same day & $1.99(0.77-5.17)$ & $3.47(1.27-9.47)$ & $4.74(1.24-18.13)$ & II.I7 (2.65-47.15) \\
\hline In a 7-day period & $2.37(1.05-5.32)$ & $3.13(1.26-7.78)$ & $4.84(1.54-15.19)$ & $9.85(2.85-34.02)$ \\
\hline In a 14-day period & $2.30(1.13-4.67)$ & $3.02(1.31-6.96)$ & $4.02(1.49-10.87)$ & $8.96(3.01-26.67)$ \\
\hline In a 30-day period & $2.03(1.07-3.84)$ & $3.01(1.45-6.25)$ & $3.67(1.59-8.48)$ & $9.67(3.8 \mathrm{I}-24.59)$ \\
\hline
\end{tabular}

Notes: $\mathrm{N}$ includes cases and matched controls from the respective subgroup. ${ }^{2} \mathrm{Adjusted}$ for: hypertension, heart failure, diabetes, chronic kidney disease, care level and

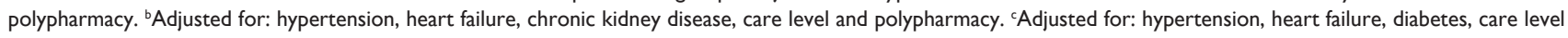

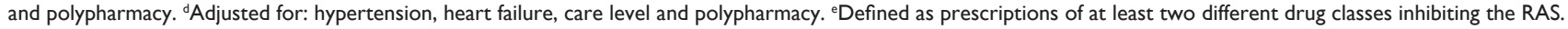
Abbreviation: RAS, renin-angiotensin system. 
AKI could be found in patients with diabetes and/or chronic kidney disease irrespective of the underlying definition of dual RAS blockade and in the sensitivity analyses for the overall cohort.

In general, our results are in line with a retrospective, cross-sectional study, which considered antihypertensive prescribing in four German nursing homes. ${ }^{22}$ However, the authors of that study only included patients with a diagnosis of arterial hypertension. The authors of another recent study found that prevalence and incidence of dual RAS blockade declined in line with recommendations. ${ }^{23}$ However, their study included a longer study period than we did in our study. The authors of a study based on an Irish primary care reimbursement services database reported that co-prescribing of ACEIs and ARBs did not appear to be influenced by results from major clinical trials. ${ }^{24}$ Further, ACEIs and ARBs were more likely to be co-prescribed in subgroups of the population with diabetes, hypertension and heart failure. Earlier, between 2000 and 2009, an increase in the prescribing of a dual RAS blockade with ACEIs and ARBs was observed. ${ }^{24}$

The definition of a dual RAS blockade is crucial and to a certain extent assumptions had to be made. The previously mentioned studies from the UK and Ireland also used the same-day definition that we employed. The definition of co-prescribing on the same day appears to be the most conservative approach and is likely to have high specificity in identifying patients undergoing dual RAS inhibition. It further should provide the highest probability that the dual RAS blockade was intended by the prescribing physician; however, it may underestimate the overall extent of coprescribing. Our sensitivity analyses used wider time windows to estimate the extent of concomitant prescribing, with a 30-day window representing the upper limit. As was to be expected, similar trends were observed but with higher prevalences than the same-day definition.

The safety warnings of the EMA in 2012 included concerns of hyperkalemia, hypotension and AKI with dual RAS blockade compared with the use of one drug alone. Due to the character of the underlying claims data, we restricted our analyses to the examination of a potential association with AKI, since hyperkalemia and low blood pressure are usually secondary diagnoses and not coded in hospital main discharge diagnoses as the reason for hospitalization.

Irrespective of mono or combination therapy, drugassociated AKI is known to be a common cause of potential adverse drug event related to the use of ACEIs and ARBs..$^{25,26}$ We found an association of dual RAS blockade with AKI in the subgroup analyses, but, except for the sensitivity analyses, not in the overall study cohort, therefore confirming the vulnerable subpopulations addressed by the EMA recommendations. This is important when assessing the individual benefits and disadvantages of dual RAS blockade and making clinically meaningful decisions. However, although we found an association of dual RAS blockade with AKI in patients with diabetes and/or chronic kidney disease, we were not able to assess the individual risk-benefit ratio. As an exception and in individual cases, use of candesartan or valsartan as add-on therapy to an ACEI in patients with heart failure may be recommended to be carried out under supervision of specialists with close monitoring of renal function, electrolytes and blood pressure. ${ }^{7}$ However, we were not able to assess the appropriateness of such a treatment.

Some strengths and limitations of our study need to be considered. The study was conducted using claims data from a large statutory health insurance comprising data on about six million insurants, which corresponds to more than seven percent of the German population. It thus provides data on the practice of drug prescribing in a real-life setting on a population level. Since data are available with the exact date of drug prescription, there is low potential for misclassification of drug exposure when compared with field studies based on interview data. However, we did not have information about whether patients were taking the RAS-inhibiting drugs they were prescribed. Even though we only considered confirmed outpatient diagnoses with respect to diagnostic certainty, misclassification could not be entirely ruled out, since the underlying data were originally collected for reimbursement purposes and not for scientific research. We, therefore, did not restrict our definition of chronic kidney disease (CKD) to codes referring to a decreased GFR but also more unspecific codes such as unspecified CKD. Further, misclassification of the underlying diagnoses for prescribing is possible, since no direct linkage is possible between prescriptions and diagnoses, and outpatient diagnoses in claims data usually do not have an exact date, but are only related to the quarter of a year due to 3-monthly reimbursement. However, hospital diagnoses used for consideration of potential risks are available with an exact date. Drug prescriptions issued in secondary care are not included in the data; therefore, RAS-inhibiting drugs used in the hospital setting could not be assessed, which might have led to an underestimation of the prevalence of dual RAS blockade in our study. Finally, we could not assess whether the combined use of eg, ARB with an ACEI was considered essential, nor if therapy was carried out under supervision of a specialist with close monitoring of renal function, electrolytes and blood pressure. 


\section{Conclusion}

To examine the impact of safety warnings and subsequent regulatory actions, which aim to assure that drugs are used safely, is crucial. Though the prevalence of dual RAS blockade declined over the study period, our study showed an increased risk of AKI in patients with diabetes and/or chronic kidney disease. Therefore, cautious use is indispensable in these vulnerable patients. However, risks and benefits of a dual RAS blockade have to be evaluated on an individual level.

\section{Acknowledgment}

The authors thank the DAK-Gesundheit for providing the data for this study.

\section{Disclosure}

The authors report no conflicts of interest in this work.

\section{References}

1. Makani H, Bangalore S, Desouza KA, Shah A, Messerli FH. Efficacy and safety of dual blockade of the renin-angiotensin system: meta-analysis of randomised trials. BMJ. 2013;346:f360.

2. Nakao N, Yoshimura A, Morita H, Takada M, Kayano T, Ideura T. Combination treatment of angiotensin-II receptor blocker and angiotensin-converting-enzyme inhibitor in non-diabetic renal disease (COOPERATE): a randomised controlled trial (retraction in: Lancet. 2009; 374(9697):1226). Lancet. 2003;361(9352):117-124.

3. ONTARGET Investigators, Yusuf S, Teo KK, et al. Telmisartan, ramipril, or both in patients at high risk for vascular events. N Engl J Med. 2008; 358(15):1547-1559.

4. Parving HH, Brenner BM, McMurray JJV, et al. Cardiorenal end points in a trial of aliskiren for type 2 diabetes. $N$ Engl J Med. 2012; 367(23):2204-2213.

5. European Medicine Agency. Combined use of medicines affecting the renin-angiotensin system (RAS) to be restricted - CHMP endorses PRAC recommendation [press release]. 2014. Available from: http:// www.ema.europa.eu/ema/index.jsp?curl=pages/news_and_events/ news/2014/05/news_detail_002107.jsp\&mid=WC0b01ac058004d5c1. Accessed March 7, 2018.

6. McMurray JJV, Krum H, Abraham WT, et al. Aliskiren, enalapril, or aliskiren and enalapril in heart failure. $N$ Engl J Med. 2016;374(16): $1521-1532$

7. Esteras R, Perez-Gomez MV, Rodriguez-Osorio L, Ortiz A, FernandezFernandez B. Combination use of medicines from two classes of reninangiotensin system blocking agents: risk of hyperkalemia, hypotension, and impaired renal function. Ther Adv Drug Saf. 2015;6(4):166-176.

8. Pflegestatistik 2011. Pflege im Rahmen der Pflegeversicherung. Deutschlandergebnisse. Available from: www.destatis.de/DE/Publikationen/Thematisch/Gesundheit/Pflege/PflegeDeutschlandergebnisse. Accessed March 7, 2018.
9. Garg AX, Papaioannou A, Ferko N, Campbell G, Clarke JA, Ray JG. Estimating the prevalence of renal insufficiency in seniors requiring long-term care. Kidney Int. 2004;65(2):649-653.

10. Joseph J, Koka M, Aronow WS. Prevalence of moderate and severe renal insufficiency in older persons with hypertension, diabetes mellitus, coronary artery disease, peripheral arterial disease, ischemic stroke, or congestive heart failure in an academic nursing home. J Am Med Dir Assoc. 2008;9:257-259.

11. Reuther S, van Nie N, Meijers J, Halfens R, Bartholomeyczik S. [Malnutrition and dementia in the elderly in German nursing homes. Results of a prevalence survey from the years 2008 and 2009]. Z Gerontol Geriatr. 2013;46(3):260-267. German.

12. Meyer G, Köpke S, Haastert B, Mühlhauser I. Restraint use among nursing home residents: cross-sectional study and prospective cohort study. J Clin Nurs. 2009;18(7):981-990.

13. Richter T, Mann E, Meyer G, Haastert B, Köpke S. Prevalence of psychotropic medication use among German and Austrian nursing home residents: a comparison of 3 cohorts. J Am Med Dir Assoc. 2012; 13(2):187.e7-187.e13.

14. Gordon AL, Franklin M, Bradshaw L, Logan P, Elliott R, Gladman JRF. Health status of UK care home residents: a cohort study. Age Ageing. 2014;43(1):97-103.

15. Dörks M, Herget-Rosenthal S, Schmiemann G, Hoffmann F. Polypharmacy and renal failure in nursing home residents: results of the inappropriate medication in patients with renal insufficiency in nursing homes (IMREN) study. Drugs Aging. 2015;33(1):45-51.

16. Schmidt I, Claesson CB, Westerholm B, Svarstad BL. Resident characteristics and organizational factors influencing the quality of drug use in Swedish nursing homes. Soc Sci Med. 1998;47(7):961-971.

17. Snowdon J, Day S, Baker W. Audits of medication use in Sydney nursing homes. Age Ageing. 2006;35(4):403-408.

18. Furniss L, Craig SK, Burns A. Medication use in nursing homes for elderly people. Int J Geriatr Psychiatry. 1998;13(7):433-439.

19. Hoffmann F, Kaduszkiewicz H, Glaeske G, van den Bussche H, Koller D. Prevalence of dementia in nursing home and community-dwelling older adults in Germany. Aging Clin Exp Res. 2014;26(5):555-559.

20. Stokes JA, Purdie DM, Roberts MS. Factors influencing PRN medication use in nursing homes. Pharm World Sci. 2004;26(3):148-154.

21. Altman D, Machin D, Bryant T GM. Statistics with Confidence. 2nd ed. London: BMJ Books; 2000.

22. Lochner S, Kirch W, Schindler C. Managing hypertension among nursing-home residents and community-dwelling elderly in Germany: a comparative pharmacoepidemiological study. Eur J Clin Pharmacol. 2012;68(5):867-875.

23. Allen C, Donegan K. The impact of regulatory action on the coprescribing of renin-angiotensin system blockers in UK primary care. Pharmacoepidemiol Drug Saf. 2017;26(7):858-862.

24. Wan Md Adnan WA, Zaharan NL, Bennett K, Wall CA. Trends in coprescribing of angiotensin converting enzyme inhibitors and angiotensin receptor blockers in Ireland. Br J Clin Pharmacol. 2011;71(3):458-466.

25. Handler SM, Cheung PW, Culley CM, et al. Determining the incidence of drug-associated acute kidney injury in nursing home residents. $J \mathrm{Am}$ Med Dir Assoc. 2014;15(10):719-724.

26. Marcum ZA, Amuan ME, Hanlon JT, et al. Prevalence of unplanned hospitalizations caused by adverse drug reactions in older veterans. J Am Geriatr Soc. 2012;60(1):34-41.
Clinical Interventions in Aging

\section{Publish your work in this journal}

Clinical Interventions in Aging is an international, peer-reviewed journal focusing on evidence-based reports on the value or lack thereof of treatments intended to prevent or delay the onset of maladaptive correlates of aging in human beings. This journal is indexed on PubMed Central, MedLine,

\section{Dovepress}

CAS, Scopus and the Elsevier Bibliographic databases. The manuscript management system is completely online and includes a very quick and fair peer-review system, which is all easy to use. Visit http://www.dovepress. com/testimonials.php to read real quotes from published authors. 